\title{
Ultrasound Image Segmentation Based On Probability Distance and Maximum Likelihood
}

\author{
Bo Liu ${ }^{1}$ H. D. Cheng ${ }^{1,2}$ Jianhua Huang ${ }^{1}$ Jiafeng Liu ${ }^{1}$ \\ ${ }^{1}$ School of Computer, Harbin Institute of Technology, Harbin, China \\ ${ }^{2}$ Department of Computer Science, Utah State University, Logan, UT 84322 U.S.A
}

\begin{abstract}
In this paper, a novel geometric active contour model for segmenting ultrasound image is presented. A partial differential equation is designed to minimize the difference between the actual and the estimated intensity probability distributions of the image regions. The Rayleigh distribution and maximum likelihood method are applied for the estimation of the intensity distributions of the regions. The approximation of the flow is based on finite difference with a non reinitialization strategy. The experimental results demonstrate that the proposed approach can segment images accurately and effectively.
\end{abstract}

Keywords: active contour, level set, segmentation, ultrasound, probability distance

\section{Introduction}

The segmentation of ultrasound images is a very difficult task because ultrasound images often have low contrast and blurry boundaries between the objects and background, and have a low signal/noise ratio. The details of ultrasound images are greatly reduced due to the speckle noise.

Many methods have been proposed to segment ultrasound images. These methods can be divided into the following categories: Markov random fields (MRF)
[1], watershed [2], region growing [3], cell-competition [4], active contours [3], [5], etc.

Several active contour and level set methods have been proposed to segment ultrasound images [3, 5]. The level set method was proposed by Osher and Sethian [6]. Level set active contour models can be divided into two classes: "edge-driven" and "region-driven". The "edge-driven" approach [7] is to track the boundaries between regions by the precomputed edge indicators, and when the evolving equation reaches a steady state, the boundary curve embedded in the zero level set has the maximum edge indicator value. The "region-driven" model was discussed in [8].

In this paper, a novel active contour model is proposed. The model is formulated based on minimizing the differences between actual and estimated intensity distributions in both inside and outside regions, respectively. In the proposed method, the prior knowledge and maximum likelihood method was integrated into the active contour model based on the probability distance, and the estimated intensity distributions are computed automatically and adaptively during the evolution of the level set function.

\section{Active contour based on probability} distance and maximum likelihood

\subsection{Segmentation criterion}


Consider image $I$ as a real positive function defined in a domain $\Omega \subset R^{2}$. The inner and outer regions of the objects can be defined as $\Omega_{i}$ and $\Omega_{e}$, and $\Omega_{i} \cup \Omega_{e}=\Omega, \Omega_{i} \cap \Omega_{e}=\phi$. Suppose the intensity distributions of the both regions have a prior form, and the distributions can be estimated by using the actual intensities of the two regions. Then, the actual intensity distributions of $\Omega_{i}$ and $\Omega_{e}$ can be defined as $P_{i}$ and $P_{e}$, and estimated intensity probability distributions of $\Omega_{i}$ and $\Omega_{e}$ can be define as $P_{i}^{E}$ and $P_{e}^{E}$. The differences between the actual probability distributions and the estimated probability distributions can be measured by some probability distance metrics, for instance, the discrete Kullback-Leibler divergence (KL-divergence):

$$
d\left(P, P^{E}\right)=\sum_{i=0}^{255} P(i)\left(\log P(i) / \log P^{E}(i)\right)
$$

However, as the KL-divergence is not symmetric, in this paper, the mean square distance is employed:

$$
D\left(P, P^{E}\right)=\sum_{i=0}^{255} P(i)\left(P(i)-P^{E}(i)\right)^{2}
$$

The criterion of the proposed segmentation method becomes: to find a boundary pixel set $C$ that partitions the ultrasound image into two kinds of regions: $\Omega_{i}$ and $\Omega_{e}$, and makes the total difference of the probability distributions of the two regions minimum, i.e., this process can be presented as finding the minimum of the energy function:

$$
E_{D}(C)=D\left(P_{i}, P_{i}^{E}\right)+D\left(P_{e}, P_{e}^{E}\right)
$$

Let $A_{i}$ and $A_{e}$ be the number of the pixels in inner and outer regions $A_{i}$ and $A_{e}$ can be computed as below:

$$
\begin{aligned}
& A_{i}=\iint_{\Omega_{i}} d x d y \\
& A_{e}=\iint_{\Omega_{e}} d x d y
\end{aligned}
$$

The actual distributions $P_{i}$ and $P_{e}$ can be computed:

$$
\begin{aligned}
P_{i}(g) & =\frac{\iint_{\Omega_{i}} \delta(I(x, y)-g) d x d y}{A_{i}}, g \in[0,255] \\
P_{e}(g) & =\frac{\iint_{\Omega_{e}} \delta(I(x, y)-g) d x d y}{A_{e}}, g \in[0,255]
\end{aligned}
$$

where $\delta(\bullet)$ is the Dirac function, $g$ is the intensity level and $I(x, y)$ is the gray intensity in position $(\mathrm{x}, \mathrm{y})$.

It has been stated in [9] that, the intensity distributions of different tissues can be considered as Rayleigh distributions with different parameters $\sigma^{2}$ :

$$
P_{\text {Rayleigh }}\left(I, \sigma^{2}\right)=\left(I / \sigma^{2}\right) \cdot \exp \left(-I^{2} / 2 \sigma^{2}\right)
$$

The likelihood function of each region can be formulated as:

$$
L_{j}=\prod_{(x, y) \in A_{j}} P_{\text {Rayleigh }}\left(I(x, y), \sigma_{j}^{2}\right)
$$

Using maximum likelihood method, the parameters of both inner and outer regions maximizing the likelihood functions can be calculated as [5]:

$$
\sigma_{j}^{2}=\frac{\iint_{\Omega_{j}} I^{2}(x, y) d x d y}{2 A_{j}}
$$

where the subscript $j \in\{i, e\}$. Then $P_{i}^{E}$ and $P_{e}^{E}$ can be calculated and the 
energy function can be computed if the boundary pixel set $C$ is given.

The importance of the two probability distances in $E_{D}$ is considered as equal. To solve such problem, $A_{i}$ and $A_{e}$ are integrated into the energy function as the weights:

$$
E_{P}(C)=A_{i} \cdot D\left(P_{i}, P_{i}^{E}\right)+A_{e} \cdot D\left(P_{e}, P_{e}^{E}\right)
$$

To prevent over-segmentation, a regular term should be introduced. According to Mumford-Shah model [8], a length term is introduced into the energy function, and the energy function becomes:

$$
E(C)=\alpha \cdot \text { length }(C)+\beta \cdot E_{P}(C)
$$

where $\alpha$ and $\beta$ are the weights of the energies.

\subsection{Level set implementation}

Integrate the actual and estimated distributions and the boundary into the energy function and the energy function can be transformed as:

$$
\begin{array}{r}
E(C)=\alpha \cdot \int_{C} d x d y+\beta \cdot\left(\iint_{\Omega_{i}}\left(p_{i}-p_{i}^{E}\right)^{2} d x d y\right. \\
\left.+\iint_{\Omega_{e}}\left(p_{e}-p_{e}^{E}\right)^{2} d x d y\right)
\end{array}
$$

A auxiliary function $\phi:(0, \infty) \times \Omega \rightarrow R$ is introduced into Eq.(13). Let $\Omega_{i}$ and $\Omega_{e}$ be the set $\{(x, y) \mid \phi(x, y)>0,(x, y) \in \Omega\}$ and set $\{(x, y) \mid \phi(x, y)<0,(x, y) \in \Omega\}$ respectively, then the set $C$ can be defined implicitly by the zero level set of $\phi$. Using the heavyside function:

$$
H(x)=\left\{\begin{array}{ll}
1 & \text { if } x \geq 0 \\
0 & \text { if } x<0
\end{array}, \frac{\partial H(x)}{\partial x}=\delta(x)\right.
$$

the energy function can be rewritten as:

$$
\begin{aligned}
& E\left(\Omega_{i}, \Omega_{e}\right)=\alpha \cdot \int_{\Omega}|\nabla H(\phi(x, y))| d x d y \\
& \quad+\beta \cdot \iint_{\Omega}\left(p_{i}-p_{i}^{E}\right)^{2} H(\phi(x, y)) d x d y \\
& \quad+\beta \cdot \iint_{\Omega}\left(p_{e}-p_{e}^{E}\right)^{2}(1-H(\phi(x, y))) d x d y
\end{aligned}
$$

Then the gradient flow can be derived:

$$
\begin{aligned}
& \frac{\partial \phi}{\partial t}=\delta(\phi)\left[\begin{array}{l}
\alpha \cdot \operatorname{div}\left(\frac{\nabla \phi}{|\nabla \phi|}\right) \\
-\beta \cdot\left(p_{i}-p_{i}^{E}\right)^{2}+\beta \cdot\left(p_{e}-p_{e}^{E}\right)^{2}
\end{array}\right] \\
& \phi(0, x, y)=\phi^{0}(x, y) \text { in } \Omega \\
& \frac{\delta(\phi)}{|\nabla \phi|} \cdot \frac{\partial \phi}{\partial \vec{n}}=0 \text { on } \partial \Omega
\end{aligned}
$$

where $\phi^{0}$ is the initial level set function, and the last equation in Eq. (16) is the boundary condition.

\section{Numerical Approximation}

To approximate Eq.(16), the heavyside function must be approximated first. In this paper, the approximation of heavyside function is same as that in [8]:

$$
H_{\varepsilon}(z)=\frac{1}{2}\left(1+\frac{2}{\pi} \arctan \left(\frac{z}{\varepsilon}\right)\right)
$$

The value of parameter $\varepsilon$ used in this paper is 1.5 .

In this paper, a strategy without reinitialization [10] is applied. A penalizing term was introduced into the energy function; the penalizing term is [10]:

$$
E_{R}(\phi)=\iint_{\Omega} \frac{1}{2}(|\nabla \phi|-1)^{2} d x d y
$$

The energy function becomes:

$$
E(C)=\alpha \cdot \text { length }(C)+\beta \cdot E_{P}(C)+\gamma \cdot E_{R}(\phi)
$$

The gradient flow can be re-written as: 


$$
\begin{aligned}
\frac{\partial \phi}{\partial t}=\delta(\phi) & {\left[\begin{array}{l}
\alpha \cdot \operatorname{div}\left(\frac{\nabla \phi}{|\nabla \phi|}\right) \\
-\beta \cdot\left(p_{i}-p_{i}^{E}\right)^{2}+\beta \cdot\left(p_{e}-p_{e}^{E}\right)^{2}
\end{array}\right] } \\
& +\gamma \cdot\left(\Delta \phi-\operatorname{div}\left(\frac{\nabla \phi}{|\nabla \phi|}\right)\right)
\end{aligned}
$$

The right hand side of Eq. (20) can be approximated simply by using the central difference instead of the up-wind difference [10]. The complete approximation is:

$$
\begin{aligned}
& K_{i, j}= D_{i, j}^{0 \times j} \cdot\left(\frac{D_{i, j}^{0 \times} \phi}{\sqrt{\left(D_{i, j}^{0 \times} \phi\right)^{2}+\left(D_{i, j}^{0.0} \phi\right)^{2}}}\right) \\
&+ D_{i, j}^{0 y} \cdot\left(\frac{D_{i, j}^{0 y} \phi}{\sqrt{\left(D_{i, j}^{0 \times} \phi\right)^{2}+\left(D_{i, j}^{0 y} \phi\right)^{2}}}\right) \\
& L_{i, j}= \Delta \phi=D_{i, j}^{0 \times x} \phi+D_{i, j}^{0 y y} \phi \\
& \frac{\phi_{i, j}^{\phi+1}-\phi_{i, j}^{p}}{\Delta t}=\delta_{\varepsilon}(\phi)\left[\begin{array}{l}
\alpha \cdot K_{i, j} \\
-\beta \cdot\left(p_{i}-p_{i}^{E}\right)^{2}+\beta \cdot\left(p_{e}-p_{e}^{E}\right)^{2}
\end{array}\right] \\
&+\gamma \cdot\left(L_{, j}-K_{i, j}\right)
\end{aligned}
$$

where $\mathrm{D}^{0}$ is central difference operator.

Finally, the steps of the proposed algorithm are:

- Initialize the level set function $\phi$ by $\phi^{0}$

- Compute the actual and estimated probability distributions of the inner and outer regions using Eq.(6), Eq.(7) and Eq.(10)

- Using Eq.(23) to compute $\phi^{n+1}$ from $\phi^{n}$

- Check the convergence of $\phi$, if it still does not reach the steady state, continue the evolution.

\section{Experimental Result}

To validate the proposed method, we conduct experiments on $40 \mathrm{~B}$-mode ultrasound images, which are from the database in [11]. We have chosen different weight parameters in the proposed active contour model. If the length weight, $\alpha$, is low, more small size objects can be detected, and the model emphasizes on the image features; on the other hand, if it is high, a smoother boundary curve can be generated. In the experiments, $\alpha=3.0$. The weight of region term $\beta$ should be proportional to the size of image. The value $\beta$ can be written as $A \cdot b$, where $A$ is the size of the image, and $b$ is the weight parameter, which is 0.1 in the experiments. The weight of penalizing term $\gamma$ is inversely proportional to the timestep $\Delta t$, and the product of $\gamma$ and $\Delta t$ should be less than 0.25 for the stability [10]. The timestep $\Delta t$ is selected as 3.0 in this paper, and $\gamma=0.2 / 3.0$.

The initial level set function $\phi^{0}$ is set following the method in [10]:

$$
\phi_{x, y}^{0}=\left\{\begin{array}{cc}
-c_{0} & (\mathrm{x}, \mathrm{y}) \in \Omega_{0}-\partial \Omega_{0} \\
0 & (\mathrm{x}, \mathrm{y}) \in \partial \Omega_{0} \\
c_{0} & (\mathrm{x}, \mathrm{y}) \in \Omega-\Omega_{0}
\end{array}\right.
$$

where $\Omega_{0}$ is a subset in the image domain $\Omega$, and $\partial \Omega_{0}$ is the boundary of $\Omega ; C_{0}=4$ in this paper.

To validate the proposed method, the regions of interest (ROIs) selected by this method is compared with the ROIs manually selected by the radiologists. Three area error metrics: the true positive ratio (TP), the false positive ratio (FP), and the false negative ratio (FN) are calculated. Let $A_{a}$ be the ROIs determined by the proposed method and $A_{m}$ be the ROIs manually determined by the radiologists, then

$$
T P=\frac{\left|A_{m} \cap A_{a}\right|}{A_{m}}
$$


$F N=\frac{\left|A_{m} \cup A_{a}-A_{a}\right|}{A_{m}}$
$F P=\frac{\left|A_{m} \cup A_{a}-A_{m}\right|}{A_{m}}$

The values of the three metrics are listed in Table. 1.

The convergence of the evolution of the level set function in the proposed method is very fast, in most of the cases, it can reach the convergence in less than 300 iterations, and the evaluating boundary can reach the real boundary even in less than 100 iterations in many cases.

An example of the evolution process of the proposed method is shown in Fig.1. The ROI finally segmented out is shown in Fig.2(a); the result of the same image using method in [8] is also shown in Fig.2(b) for comparing, where are many trivial regions, i.e., it is over-segmented. In addition, the boundary got by the proposed method is much smoother. Fig.3 shows another example to manifest the effectiveness of the proposed approach.

The speed of the method in [8] is much slower than that of the proposed method, too. The experiments were done on a 3.2 $\mathrm{GHz}$ Pentium 4 PC with 1GB RAM using MATLAB. The average CPU time of the proposed method and method in [8] are listed in table. 2

Table1. Performance

\begin{tabular}{|l|l|l|}
\hline TP Ratio & FP Ratio & FN Ratio \\
\hline $90.12 \%$ & $9.88 \%$ & $10.54 \%$ \\
\hline
\end{tabular}

Table2. Average CPU time (second)

\begin{tabular}{|c|c|}
\hline Proposed Method & Method in [8] \\
\hline $38.72 \mathrm{~s}$ & $403.1 \mathrm{~s}$ \\
\hline
\end{tabular}

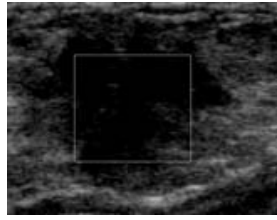

(a)

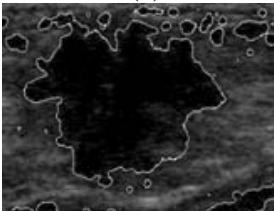

(c)

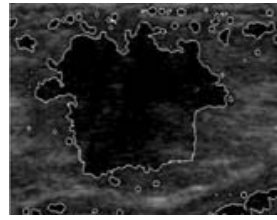

(b)

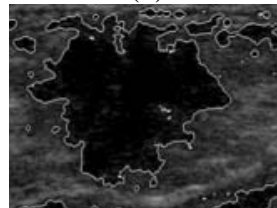

(d)
Fig.1: The evolution of the boundary curve c.

(a) The initial contour; (b) After 5 iterations;

(c) After 10 iterations; (d) After 60 iterations

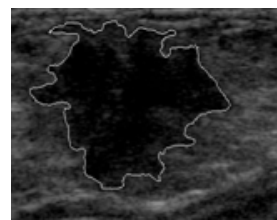

(a)

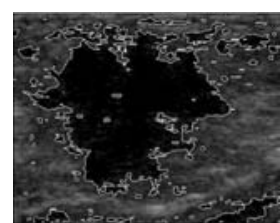

(b)
Fig.2: (a) The final segmented ROI (converged in 150 iterations); (b) The result using the method in [8] (converged in 1300 iterations).

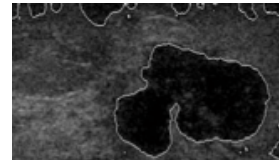

(a)

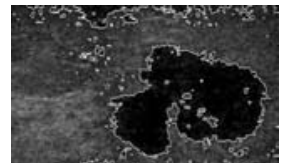

(b)
Fig.3: Another example. (a) The segmentation of proposed method (converged in 250 iterations); (b) The result using the method in [8] (converged in 1800 iterations).

\section{Conclusions}

In this paper, a segmentation method for ultrasound image based on active contour and the prior knowledge of the intensity distribution is proposed. The proposed method can accurately segment the ultrasound images with a quite fast speed. 
And it is robust to the noise. It is very useful for CAD systems.

\section{Acknowledgement}

This work is supported, in part, by National Science Foundation of China; grant numbers: $\quad 60573071$; 60873142; 30670546

\section{References}

[1] D. Boukerroui, et al., "Segmentation of ultrasound images multiresolution 2D and 3D algorithm based on global and local statistics," Pattern Recognition Lett., vol. 24, pp. 779-790, 2003.

[2] Y. L. Huang and D. R. Chen, "Watershed segmentation for breast tumor in 2-D sonography," Ultrasound Med. Biol., vol. 30, no. 5, pp. 625-632, May 2004.

[3] A. Madabhushi and D. N. Metaxas, "Combining low-, high-level and empirical domain knowledge for automated segmentation of ultrasonic breast lesions," IEEE Trans. Med. Imag., vol. 22, no. 2, pp. 155169, Feb. 2003.

[4] C. M. Chen, Y. H. Chou, C. S. K. CHEN, "Cell-Competition: a new segmentation algorithm for multiple objects with irregular boundaries in ultrasound images," Ultrasound Med. Biol., vol. 31, no. 12, pp. 1647-1664, Dec. 2005.
[5] A. Sarti, C. Corsi, E. Mazzini, and C. Lamberti, "Maximum Likelihood Segmentation of Ultrasound Images with Rayleigh Distribution," IEEE Trans. Ultrasonics, Ferroelectrics, and Frequency Control, vol. 52, no. 6, June 2005.

[6] S. Osher and J. A. Sethian, "Front propagating with curvature dependent speed: Algorithms based on Hamilton Jacobi formulation,” $J$. Comput. Phys., vol. 79, pp. 12-49, 1988.

[7] V. Caselles, R. Kimmel, and G. Sapiro, "On geodesic active contours,” Int. J. Comput. Vis., vol. 22, no. 1, pp. 61-79, 1997.

[8] T. F. Chan and L. A. Vese, "Active contours without edges," IEEE Trans. Image Processing, vol. 10, no. 2, pp. 266- 277, 2001.

[9] R.F.Wagner, S. W. Smith, J.M. Sandrik, and H. Lopez, "Statistics of speckle in ultrasound B-scans,” IEEE Trans. Sonics Ultrason., vol. 30, no. 3, pp. 156 - 163, 1983.

[10] C. M. Li, C. Y. Xu, C. F. Gui, M. D. Fox, "Level set evolution without reinitialization: a new variational formulation”, 2005 IEEE Comp. Soc. Conf., pp. 1063-1069, San Diego, USA, 2005.

[11] Y. H. Guo, H. D. Cheng, J. H. Huang, J. W. Tian, W. Zhao, L. T. Sun, and Y. X. Su, "Breast ultrasound image enhancement using fuzzy logic," Ultrasound Med. Biol., vol. 32, no. 2, pp. 237-247, 2006 